\title{
A Facile Route to a Novel Aza-Crown Ether Incorporating Three Thiophene Moieties.
}

\author{
Joan Halfpenny*, Phillip B. Rooney and Zachary S. Sloman.
}

Department of Chemistry and Physics, Nottingham Trent University, Clifton Lane, Nottingham NG11 8NS, UK

*Corresponding author. Fax: (+44) 0115 9486636; e-mail joan.halfpenny@ntu.ac.uk

\begin{abstract}
The preparation of the first of a novel type of large thiophene-containing aza-crown ether is reported. The macrocycle is synthesised by linking a 3,4-dialkoxythiophene moiety with two 3-hydroxythiophene units and ring closure is effected by reaction with piperazine via the Mannich reaction.
\end{abstract}

Keywords Aza-crown, Thiophene, Macrocycle, Mannich reaction.

The syntheses of non-aromatic and benzene-containing aza-crown ethers have been widely reported, ${ }^{1}$ however thiophene analogues are poorly represented in the literature. ${ }^{2}$ Aza-crown ethers have complexing properties which are intermediate between those of crown ethers and those of nitrogen donor macrocycles. They exhibit enhanced coordinating ability towards ammonium salts and transition metals over crown ethers ${ }^{3,4}$ as well as having important applications as synthetic receptors in molecular recognition processes. ${ }^{5}$ We report in this paper a new route to the first of a novel type of large aza-crown ether incorporating three thiophene rings. The method of ring closure employed utilises the Mannich reaction which gives selective substitution of the 3-alkoxythiophene units over the 3,4-dialkoxythiophene unit. The synthetic route is shown in Scheme 1.

To diethyl 3,4-dihydroxythiophene-2,5-dicarboxylate, 1, (1 eq) and anhydrous potassium carbonate $(1.2 \mathrm{eq})$ in DMF $\left(1.0 \mathrm{~cm}^{3} \mathrm{mmol}^{-1}\right)$ heated at $100^{\circ} \mathrm{C}$ was added 5-tosyloxy-3oxapentan-1-ol (1.2 eq) in DMF $\left(0.5 \mathrm{~cm}^{3} \mathrm{mmol}^{-1}\right)$ by means of a syringe pump. After the addition heating was continued for 12 hours. The solvent was removed in vacuo and the crude mixture was partitioned between water and EtOAc. The organic layer was dried $\left(\mathrm{MgSO}_{4}\right)$ and evaporated. Diethyl 3,4-bis(5'-hydroxy-3'-oxapentyloxy)thiophene-2,5dicarboxylate, 2 , was obtained in $56 \%$ yield $^{6}$ as an oil after column chromatography (silica; EtOAc). To crude 2 (5.10 g, $0.012 \mathrm{~mol})$, in dry DCM $\left(45 \mathrm{~cm}^{3}\right)$ and triethylamine $\left(10 \mathrm{~cm}^{3}\right)$, was added methanesulfonyl chloride $(8.04 \mathrm{~g}, 0.07 \mathrm{~mol})$ with ice cooling. The mixture was stirred at room temperature for 10 minutes, then poured into water $\left(100 \mathrm{~cm}^{3}\right)$. The organic layer was separated, washed with water, dried $\left(\mathrm{Na}_{2} \mathrm{SO}_{4}\right)$ and evaporated. The resulting oil was purified by column chromatography, (silica; EtOAc-light petroleum, 2:1), to give diethyl 3,4-bis(5'-methanesulfonyloxy-3'-oxapentyloxy)thiophene-2,5-dicarboxylate, 3, as an oil, (3.22 g, 47\% yield). ${ }^{7}$ To add the end thiophene units, 3 ( $2.14 \mathrm{~g}, 3.64 \mathrm{mmol}$ ) was added to a 
suspension of methyl 3-hydroxythiophene-2-carboxylate (1.25 g, $8.23 \mathrm{mmol}$ ) and potassium carbonate $(1.50 \mathrm{~g}, 10.87 \mathrm{mmol})$ in DMF $\left(50 \mathrm{~cm}^{3}\right)$ and stirred and heated $\left(100^{\circ} \mathrm{C}\right)$ for 12 hours. Upon cooling, the inorganic material was filtered off and the filtrate was evaporated to dryness in vacuo. The resulting oil was dissolved in EtOAc $\left(75 \mathrm{~cm}^{3}\right)$ and washed with water. The organic layer was dried $\left(\mathrm{MgSO}_{4}\right)$, and evaporated to give an oil, which was subjected to column chromatography, (silica; EtOAc-light petroleum, 3:1), to give diethyl 3,4-bis[5'-(2"methoxycarbonyl-3"-thienyloxy)-3'-oxapentyloxy]-thiophene-2,5-dicarboxylate, 4, (1.93 g, 75\%). ${ }^{8}$ Having successfully coupled the three thiophene moieties, the tetraester, 4 , was stirred with a $30 \%$ excess of sodium hydroxide solution (2M) and an equal volume of ethanol at $60^{\circ} \mathrm{C}$ for 1 hour. The cooled solution was acidified with hydrochloric acid (4M), and the subsequent slurry was stirred for a further hour. The ethanol was removed in vacuo and the precipitate filtered off and recrystallised from water to afford 3,4-bis[5'-(2"-carboxy-3"thienyloxy)-3'-oxapentyloxy] thiophene-2,5-dicarboxylic acid, 5, as a white solid, (92\%). ${ }^{9}$ In order to decarboxylate the tetraacid, compound 5, quinoline $\left(3 \mathrm{~cm}^{3} \mathrm{~g}^{-1}\right)$ and copper(I) oxide ( 0.25 mol per mol of $\mathrm{CO}_{2}$ expected) were heated $\left(180^{\circ} \mathrm{C}\right)$ for 45 minutes under nitrogen. Upon cooling, the mixture was filtered and the residue was washed with diethyl ether. The combined filtrate and ethereal washings were extracted with hydrochloric acid (4M), water and sodium carbonate solution (10\%). The diethyl ether layer was dried $\left(\mathrm{Na}_{2} \mathrm{SO}_{4}\right)$ and evaporated. The crude compound was purified by centrifugal chromatography, (silica; light petroleum-EtOAc, 1:1), to give 3,4-bis[5'-(3"-thienyloxy)-3'-oxapentyloxy]thiophene, 6, as a white solid (81\%). ${ }^{10}$ In the final step, 6 (0.67 g, $\left.1.47 \mathrm{mmols}\right)$ was added to glacial acetic acid $\left(50 \mathrm{~cm}^{3}\right)$, piperazine $(0.13 \mathrm{~g}, 1.51 \mathrm{mmol})$ and aqueous formaldehyde $\left(37 \%, 0.24 \mathrm{~cm}^{3}, 2.96\right.$ mmol) and stirred overnight. The solvent was removed in vacuo and the residue was basified $(4 \mathrm{M} \mathrm{NaOH})$ and extracted with dichloromethane, $\left(3 \times 20 \mathrm{~cm}^{3}\right)$. The combined organic layers were dried $\left(\mathrm{MgSO}_{4}\right)$ and evaporated to give the crude product which was purified by column chromatography, (alumina; light petroleum-EtOAc, 5;1), to give 2,5,8,14,17,20-hexaoxa27,30-diaza-11,24,33-trithia-27,30-ethylenpentacyclo[30.3. $\left.{ }^{1,32} 2{ }^{27,30} 0 .{ }^{21,25} 0 .{ }^{9,13}\right]$ pentatriaconta-1(32),9,12,21,(25),22,34-hexene, 7, as a white crystalline solid $(0.41 \mathrm{~g}, 49 \%) .{ }^{11}$

The macrocycle 7 was obtained as the sole product of the reaction. This was somewhat surprising as cyclisation should also be possible between the 2-position of the 3,4-dioxy substituted thiophene ring and the 2-position of one of the 3-oxy substituted thiophene rings. However, this latter reaction clearly does not occur as no asymmetrically substituted product was isolated from the column chromatography fractions.

\section{References}

1. Krakowiak, K. E.; Bradshaw, J. S.; Zamecka-Krakowiak, D. J. Chem. Rev. 1989, 89, 929972.

2. (a) Bailey, N. A. ; Eddy, M. M. ; Fenton, D. E. ; Jones, G. ; Moss, S. ; Mukhopadhyay, A. J. Chem. Soc. Chem. Commun. 1981, 628-630. (b) Bailey, N. A.; Eddy, M. M.; Fenton, D. E.; Moss, S.; Mukhopadhyay, A.; Jones, G. J. Chem. Soc. Dalton. Trans. 1984, 2281-2288. (c) Barker, J. M.; Chaffin, J. D. E.; Halfpenny, J.; Huddleston, P. R.; Tseki, P.F. J. Chem. 
Soc., Chem. Commun. 1993, 1733-1734. (d) Miller, D. C.; Johnson, M. R.; Ibers, J. A. J. Org. Chem. 1994, 59, 2877-2879.

3. Lehn, J. M.; Vierling, P. Tetrahedron Lett. 1980, 21, 1323-1326.

4. Izatt, R. M.; Bradshaw, J. S.; Nielson, S. A.; Lamb, J. D.; Christensen, J. J.; Sen, D. Chem. Rev. 1985, 85, 271-339.

5. Sutherland, I. O. Chem. Soc. Rev. 1986, 15, 63-91.

6. $v_{\max }\left(\right.$ film) 3426, 2937, 2872 and $1717 \mathrm{~cm}^{-1} ; \delta_{\mathrm{H}}\left(\mathrm{CDCl}_{3}\right) 1.37\left(6 \mathrm{H}, \mathrm{t}, J 7.3 \mathrm{~Hz}, \mathrm{CH}_{3}\right)$, 3.63-3.65 (4H, m, $\left.\mathrm{OCH}_{2}\right), 3.80-3.84\left(8 \mathrm{H}, \mathrm{m}, \mathrm{OCH}_{2}\right), 4.35\left(4 \mathrm{H}, \mathrm{q}, \mathrm{CO}_{2} \mathrm{CH}_{2}\right), 4.39-4.41(4 \mathrm{H}$, m, $\left.A r O C H_{2}\right) ; \delta_{\mathrm{C}}\left(\mathrm{CDCl}_{3}\right) 14.2\left(\mathrm{CH}_{3}\right), 61.5\left(\mathrm{CO}_{2} \mathrm{CH}_{2}\right), 61.7\left(\mathrm{CH}_{2} \mathrm{OH}\right), 70.2,72.9$ and 74.1 $\left(\mathrm{OCH}_{2}\right), 119.7$ (C2/C5), 153.2 (C3/C4), 160.5 (C=O); (Found: C, 49.71; H, 6.72. $\mathrm{C}_{18} \mathrm{H}_{28} \mathrm{O}_{10} \mathrm{~S}$ requires $\mathrm{C}$, 49.53; $\mathrm{H}, 6.47 \%)$

7. $v_{\max }(\mathrm{film}) 2960,2910$ and $1710 \mathrm{~cm}^{-1} ; \delta_{\mathrm{H}}\left(\mathrm{CDCl}_{3}\right) 1.37\left(6 \mathrm{H}, \mathrm{t}, J 7.3 \mathrm{~Hz}, \mathrm{O}_{2} \mathrm{CH}_{2} \underline{\mathrm{CH}}_{3}\right), 3.06$ (6H, s, $\left.\mathrm{SO}_{2} \mathrm{CH}_{3}\right), 3.80-3.87\left(8 \mathrm{H}, \mathrm{m}, \mathrm{OCH}_{2}\right), 4.34\left(4 \mathrm{H}, \mathrm{q}, J 7.3 \mathrm{~Hz}, \mathrm{CO}_{2} \mathrm{CH}_{2}\right), 4.36-4-39(8 \mathrm{H}$, $\left.\mathrm{m}, \mathrm{OCH}_{2}\right) ; \delta_{\mathrm{C}}\left(\mathrm{CDCl}_{3}\right) 14.2\left(\mathrm{CH}_{2} \mathrm{CH}_{3}\right), 37.5\left(\mathrm{SO}_{2} \mathrm{CH}_{3}\right), 61.4\left(\mathrm{CO}_{2} \underline{\mathrm{CH}_{2}}\right), 68.9,69.3,70.5$ and $73.7\left(\mathrm{OCH}_{2}\right), 119.6$ (C2/C5), 153.0 (C3/C4), 160.4 (C=O); (Found: C, 40.62; H, 5.49.

$\mathrm{C}_{20} \mathrm{H}_{32} \mathrm{O}_{14} \mathrm{~S}_{3}$ requires $\mathrm{C}$, 40.53; $\left.\mathrm{H}, 5.44 \%\right)$.

8. $v_{\max }(\mathrm{film}) 3110,2980,2950$ and $1710 \mathrm{~cm}^{-1} ; \delta_{\mathrm{H}}\left(\mathrm{CDCl}_{3}\right) 1.36\left(6 \mathrm{H}, \mathrm{t}, J 7.3 \mathrm{~Hz}, \mathrm{O}_{2} \mathrm{CH}_{2} \underline{\mathrm{CH}}_{3}\right)$, $3.81\left(6 \mathrm{H}, \mathrm{s}, \mathrm{CO}_{2} \mathrm{CH}_{3}\right)$, 3.87-3-92 (8H, m, $\left.\mathrm{OCH}_{2}\right), 4.24-4.39\left(12 \mathrm{H}, \mathrm{m}, \mathrm{CO}_{2} \mathrm{CH}_{2}\right.$ and $\left.\mathrm{OCH}_{2}\right)$, $6.85\left(2 \mathrm{H}, \mathrm{d}, J 5.6 \mathrm{~Hz}\right.$, ArH on C4), $7.37\left(2 \mathrm{H}, \mathrm{d}, J 5.6 \mathrm{~Hz}\right.$, ArH on C5); $\delta_{\mathrm{C}}\left(\mathrm{CDCl}_{3}\right) 14.2$ $\left(\mathrm{O}_{2} \mathrm{CH}_{2} \underline{\mathrm{CH}}_{3}\right), 51.6\left(\mathrm{CO}_{2} \underline{\mathrm{CH}}_{3}\right), 61.4\left(\mathrm{CO}_{2} \underline{\mathrm{CH}}_{2}\right), 69.6,70.8,71.7$ and $\left.73.8\left(\mathrm{OCH}_{2}\right), 110.0(\mathrm{C} 2)^{\prime}\right)$, 117.5 (C4'), 119.5 (C2/C5), 130.5 (C5'), 153.2 (C3/C4), 160.6 ( $\left.\underline{\mathrm{CO}}_{2} \mathrm{Et}\right), 161.1\left(\underline{\mathrm{CO}}_{2} \mathrm{Me}\right)$, 162.0 (C3'); (Found: C, 50.62; H, 4.91. $\mathrm{C}_{30} \mathrm{H}_{36} \mathrm{O}_{14} \mathrm{~S}_{3}$ requires $\mathrm{C}, 50.27 ; \mathrm{H}, 5.06 \%$ ).

9. M.p. $150-152^{\circ} \mathrm{C}$. $v_{\max }(\mathrm{KBr}) 3100,2920,2600,1710$ and $1690 \mathrm{~cm}^{-1} ; \delta_{\mathrm{H}}\left(\mathrm{CDCl}_{3} / \mathrm{DMSO} \mathrm{d}_{6}\right)$ 3.84-3.88 (8H, m, $\left.\mathrm{CH}_{2} \mathrm{OCH}_{2}\right)$, 4.26-4.30 (4H, m, $\left.\mathrm{ArOCH}_{2}\right)$, 4.36-4.40 (4H, m, $\left.\mathrm{ArOCH}_{2}\right), 6.90$ (2H, d, $J 5.6 \mathrm{~Hz}$, ArH on C4'), 7.45 (2H, d, J 5.6 Hz, ArH on C5'), (no signal observed for $\left.\mathrm{CO}_{2} \mathrm{H}\right) ; \delta_{\mathrm{C}}\left(\mathrm{CDCl}_{3} / \mathrm{DMSO}_{-} \mathrm{d}_{6}\right) 69.3,70.3,71.4$ and $73.5\left(\mathrm{OCH}_{2}\right), 111.0\left(\mathrm{C}^{\prime}\right), 117.6\left(\mathrm{C} 4{ }^{\prime}\right)$, 120.6 (C2/C5), 130.7 (C5'), 152.3 (C3/C4), 160.4 (C3'), 162.1 and 163.2 (C=O); (Found: C, 43.25; $\mathrm{H}, 4.09 . \mathrm{C}_{24} \mathrm{H}_{24} \mathrm{O}_{14} \mathrm{~S}_{3}$ requires $\mathrm{C}$, 45.57; $\mathrm{H}, 3.82 \%, \mathrm{C}_{24} \mathrm{H}_{24} \mathrm{O}_{14} \mathrm{~S}_{3} .2 \mathrm{H}_{2} \mathrm{O}$ requires $\mathrm{C}$, 43.11; H, 4.22\%).

10. M.p. $98.5-100^{\circ} \mathrm{C} . v_{\max }(\mathrm{KBr}) 3100,3090,2920,2895,2850$ and $1575 \mathrm{~cm}^{-1} ; \delta_{\mathrm{H}}\left(\mathrm{CDCl}_{3}\right)$ 3.87-3-92 (8H, m, $\left.\mathrm{CH}_{2} \mathrm{OCH}_{2}\right)$, 4.09-4.18 (8H, m, $\left.\mathrm{ArOCH}_{2}\right)$, 6.23-6.25 (4H, m, ArH on C2/C5 and $\left.\mathrm{C2}^{\prime}\right)$, 6.75-6.78 (2H, m, ArH on $\left.\mathrm{C}^{\prime}\right)$, 7.14-7.17 (2H, m, ArH on $\left.\mathrm{C}^{\prime}\right)$; $\delta_{\mathrm{C}}\left(\mathrm{CDCl}_{3}\right)$ 69.6, 69.7, 69.9 and $70.0\left(\mathrm{OCH}_{2}\right)$, 97.6 (C2'), 98.1 (C2/C5), 119.6 (C4'), 124.7 (C5'), 147.1 (C3/C4), 157.5 (C3'); (Found: C, 52.33; H, 5.35. $\mathrm{C}_{20} \mathrm{H}_{24} \mathrm{O}_{6} \mathrm{~S}_{3}$ requires C, 52.61; H, 5.30\%). 11. M.p. $94-96^{\circ} \mathrm{C} . v_{\max }(\mathrm{KBr}) 3110,2930,2890,1570$ and $1555 \mathrm{~cm}^{-1} ; \delta_{\mathrm{H}}\left(\mathrm{CDCl}_{3}-\mathrm{DMSO}-\mathrm{d}_{6}\right)$ $2.49\left(8 \mathrm{H}, \mathrm{s}, \mathrm{NCH}_{2}\right), 3.61\left(4 \mathrm{H}, \mathrm{s}, \mathrm{ArCH}_{2} \mathrm{~N}\right), 3.81-3.90\left(8 \mathrm{H}, \mathrm{m}, \mathrm{CH}_{2} \mathrm{OCH}_{2}\right), 4.09-4.15(8 \mathrm{H}, \mathrm{m}$, $\left.\mathrm{ArOCH}_{2}\right), 6.25$ (2H, s, ArH), $6.79(2 \mathrm{H}, \mathrm{d}, J 5.3 \mathrm{~Hz}, \mathrm{ArH}$ on C4'), 7.08 (2H, d, J 5.6Hz, ArH on $\left.\mathrm{C}^{\prime}\right) ; \delta_{\mathrm{C}}\left(\mathrm{CDCl}_{3}-\mathrm{DMSO}_{6}\right) 51.8\left(\mathrm{ArCH}_{2} \mathrm{~N}\right), 52.0\left(\mathrm{NCH}_{2}\right), 69.6,70.0,70.1$ and $71.6\left(\mathrm{OCH}_{2}\right)$, 98.2 (C2/C5), 117.7 (C4'), 118.0 (C2'), 122.4 (C5'), 147.0 (C3/C4), 154.1 (C3'); (Found: C, 55.27; H, 6.16; N, 4.74. $\mathrm{C}_{26} \mathrm{H}_{34} \mathrm{~N}_{2} \mathrm{O}_{6} \mathrm{~S}_{3}$ requires C, 55. 10; $\left.\mathrm{H}, 6.05 ; \mathrm{N}, 4.94 \%\right) ;$ mle $566\left(\mathrm{M}^{+}\right.$, $100 \%)$. 


\section{Caption for Scheme 1 :}

(i) $\mathrm{K}_{2} \mathrm{CO}_{3}$, 5-tosyloxy-3-oxapentan-1-ol, DMF, $\Delta 100^{\circ} \mathrm{C}$. (ii) methanesulfonyl chloride, DCM / triethylamine, $0^{\circ} \mathrm{C}$. (iii) methyl 3-hydroxythiophene-2-carboxylate, $\mathrm{K}_{2} \mathrm{CO}_{3}$, DMF, $\Delta 100^{\circ} \mathrm{C}$.

(iv) $2 \mathrm{M} \mathrm{NaOH}_{(\mathrm{aq})}, \mathrm{EtOH}, \Delta 60^{\circ} \mathrm{C}, 4 \mathrm{M} \mathrm{HCl}$. (v) $\mathrm{Cu}_{2} \mathrm{O}$, quinoline, $\Delta 180^{\circ} \mathrm{C}$, under $\mathrm{N}_{2} \cdot$ (vi) $37 \%$ $\mathrm{CH}_{2} \mathrm{O}_{(\text {aq) }}$, piperazine, g.AcOH. 


\section{Scheme 1}

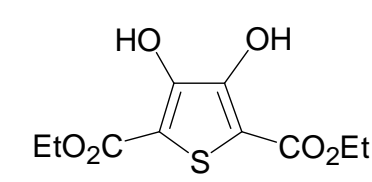

1

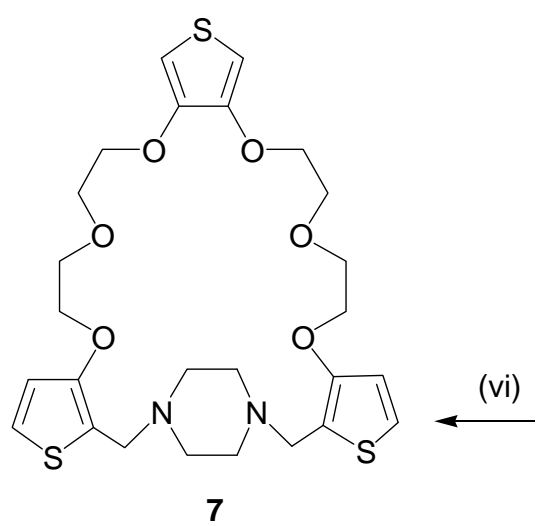

(i)

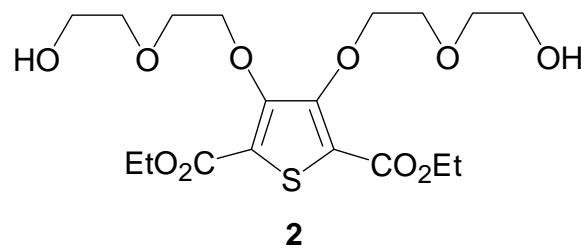

(ii)

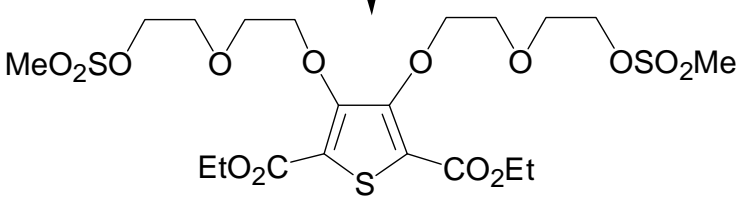

(iii)

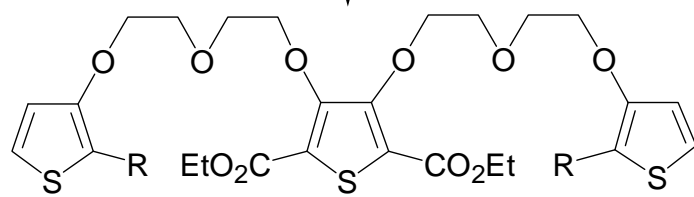

$4 \mathrm{R}=\mathrm{CO}_{2} \mathrm{Me}$

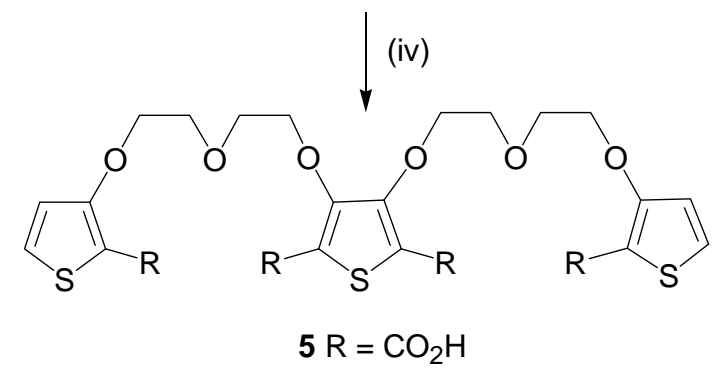

(v)

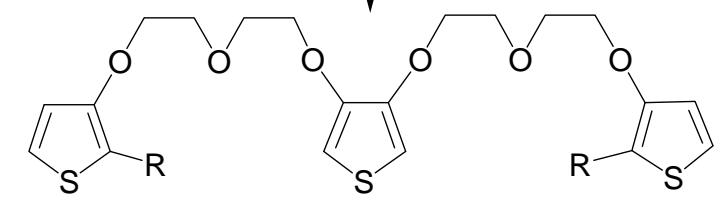

6 
A Facile Route to a Novel Aza-Crown Ether Incorporating

Three Thiophene Moieties

Joan Halfpenny ${ }^{*}$, Phillip B. Rooney and Zachary S. Sloman

Department of Chemistry and Physics, Nottingham Trent University, Clifton Lane, Nottingham NG11 8NS, UK

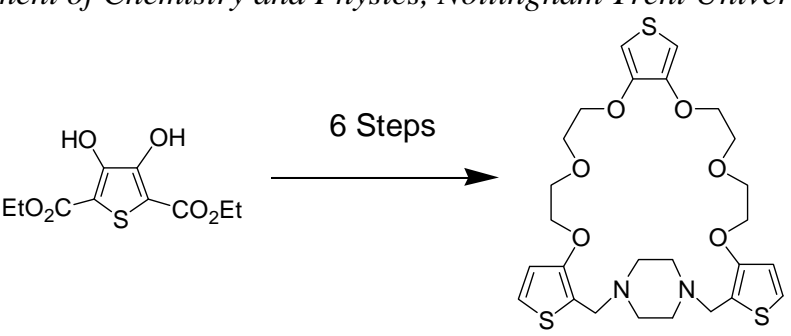

The synthesis of the first of a novel type of large thiophene-containing aza-crown ether is reported. 